\title{
OPTIMAL STRATEGIES IN GAME-CONTROL PROBLEMS OF TIMING
}

\author{
G.Klaassen', A.V.Kryazhimskii ${ }^{* *, *, 1}$, A.M.Tarasyev ${ }^{* *, *, 2}$ \\ *International Institute for Applied Systems Analysis (IIASA), \\ A-2361 Laxenburg, Austria \\ e-mail:klaassen@iiasa.ac.at,kryazhim@iiasa.ac.at,tarasiev@itasa.ac.at \\ ** Steklov Mathematical Institute of RAS, \\ Gubkina str. 8, Moscow 117966, Russia \\ e-mail:kryazhim@mi.ras.ru \\ *** Institute of Mathematics and Mechanics UrB of RAS, \\ S.Kovalevskaya str. 16, Ekaterinburg 620219, Russia \\ e-mail: tam@imm.uran.ru
}

\begin{abstract}
The paper addresses the issue of optimal investments in innovations. As an example, investments in the construction of gas pipelines are considered. Rational decisions in choosing the commercialization times (stopping times) can be associated with Nash equilibria in a game between the projects. In this game, the total benefits gained during the pipelines' life periods act as payoffs and commercialization times as strategies. The goal of this paper is to characterize multiequilibria in the game of timing. The case of two players is studied in detail. A key point in the analysis is the observation that all player's best response commercialization times concentrate at two instants that are fixed in advance. This reduces decisionmaking to choosing between two fixed investment policies, fast and slow, with the prescribed commercialization times. A description of a computational algorithm that finds all the Nash equilibria composed of fast and slow scenarios concludes the paper. Copyright (C) 2003 IFAC
\end{abstract}

Keywords: Optimal stopping problem, game of timing, multiequilibria, econometric analysis.

\section{INTRODUCTION}

If several large-scale gas pipeline projects compete for a new gas market, the choices of the commercialization times (stopping times), i.e, the times of finalizing the construction of the pipelines, determine the future structure of the market and thus become especially important. In the paper (Klaassen

\footnotetext{
${ }^{1}$ This author was supported by the Russian Fund for Fundamental Research, Grant 00-01-00682.

${ }^{2}$ This author was supported by the Russian Fund for Fundamental Research, Grant 02-01-00769, and the Program for the Sponsorship of Leading Scientific Schools, Grant 00-15-96057.
}

et al., 2001b), which motivated the present study, a detailed pipeline model based on classical patterns of mathematical economics (see (Arrow and Kurz, 1970), (Intriligator, 1971)) was designed and a best reply dynamic adaptation algorithm originating from the theory of evolutionary games (see (Friedman, 1991) - (Hofbauer and Sigmund, 1988), (Kaniovski et al., 2000), (Kryazhimskii and Osipov, 1995)(Kryazhimskii et al., 2001), (Tarasyev, 1999)) was used to estimate numerically the commercialization times for the pipeline projects competing nowadays for the Turkey gas market.

Rational choices of the commercialization times can be viewed as Nash equilibria in à game between 
the projects. We study the structure of this game in terms of game theory (see (Basar and Olsder, 1982), (Vorob'ev, 1977)). A background of the gas infrastructure model (see (Klaassen et al., 2001a)) and constructions of the optimal timing problem (see (Barzel, 1968), (Tarasyev and Watanabe, 2001)) are employed.

The model takes into account the stages of construction and exploitation of the gas pipelines. In each level, the model is optimized and estimated using appropriate techniques of theory of optimal control and theory of differential games (see (Chernousko, 1994), (Krasovskii and Subbotin, 1988), (Pontryagin et al., 1962)). At the stage of exploitation, as gas supply policies compete on market, decisionmaking is relatively clear: the competitors search for an equilibrium supply at any instant. We focus, therefore, on the stage of construction, at which investment policies compete and decisionmaking is concerned with strong long-term aftereffects. The competitors interact through choosing their commercialization times. A proper individual choice is the best response to the choices of the other competitor. Therefore, a pair of commercialization times is suitable to every competitor if and only if the commercialization time of every competitor responds best to the commercialization time of the other competitor. Such situations constitute Nash equilibria in the game under consideration. In this game, the total benefits gained during the pipelines' life periods act as payoffs and commercialization times act as strategies. Our goal is to characterize the equilibria in this game of timing. A key point in the analysis is the observation that all player's best response commercialization times concentrate at two instants that are fixed in advance. This reduces decisionmaking to choosing between two fixed investment policies, fast and slow, with the prescribed commercialization times. We describe an algorithm that finds all the Nash equilibria in the game of timing. We give results of the model-based analysis for two case studies: competing gas pipeline projects in the Caspian region, and the planned pipeline routes to the gas market in China.

\section{GAME OF TIMING}

We construct a game-theoretic model of competition of two gas pipeline projects. We associate players 1 and 2 with the investors of projects 1 and 2 , respectively. Assuming that the starting time for making investments is 0 , we consider virtual positive commercialization times of projects 1 and 2 (i.e., the final times of the construction of the pipelines), $t_{1}$ and $t_{2}$. Given a commercialization time, $t_{i}$, player $i$ ( $i=1,2)$ can estimate the cost, $C_{i}\left(t_{i}\right)$, for finalizing project $i$ at time $t_{i}$. The positive-valued cost functions $C_{i}\left(t_{i}\right)(i=1,2)$ are defined on the positive half-axis.
Assumption 2.1. For each player, $i$, the cost function, $C_{i}\left(t_{i}\right)$, is smooth, monotonically decreasing and convex.

In what follows, the rate of cost reduction for player $i$ is understood as the positive-valued monotonically decreasing function

$$
a_{i}\left(t_{i}\right)=-C_{i}^{\prime}\left(t_{i}\right)
$$

At any time $t>0$, the price of gas and costs for extraction and transportation of gas determine the benefit rate of player $1, b_{1}(t)$. The costs for extraction and transportation of gas do not depend on the state of project 2, whereas the price of gas depends on the presence of player 2 on the marketplace. Hence, the benefit rate $b_{1}(t)$ may take two values, upper $-b_{11}(t)$, and lower $-b_{12}(t)$.

$$
b_{11}(t)>b_{12}(t) .
$$

Similarly, we introduce the upper and lower benefit rates of player 2 at time $t, b_{21}(t)$ and $b_{22}(t)$,

$$
b_{21}(t)>b_{22}(t) \text {. }
$$

We assume that the positive-valued upper and lower benefit rates $b_{i 1}(t)$ and $b_{i 2}(t)(i=1,2)$ are continuous functions defined on the positive half-axis.

Assumption 2.2. For every player $i(i=1,2)$, the graph of the rate of cost reduction, $a_{i}(t)$, intersects the graph of the upper benefit rate, $b_{i 1}(t)$, from above at the unique point $t_{i}^{-}>0$, and stays below it afterwards; similarly, the graph of $a_{i}(t)$ intersects the graph of $b_{i 2}(t)$ from above at the unique point $t_{i}^{+}>0$, and stays below it afterwards.

It is clear that

$$
t_{i}^{-}<t_{i}^{+}
$$

Denote by $t_{2}$ the commercialization time of player 2 . The benefit rate of player $1, b_{1}(t)$, equals $b_{11}(t)$ for $t<t_{2}$ and equals $b_{12}(t)$ for $t \geq t_{2}$. We stress the dependence of $b_{1}(t)$ on $t_{2}$ and write $b_{1}\left(t \mid t_{2}\right)$ instead of $b_{1}(t)$

$$
b_{1}\left(t \mid t_{2}\right)=\left\{\begin{array}{l}
b_{11}(t) \text { if } t<t_{2}, \\
b_{12}(t) \text { if } t \geq t_{2} .
\end{array}\right.
$$

Similarly, a commercialization time $t_{1}$ of project 1 determines the benefit rate of player 2 as

$$
b_{2}\left(t \mid t_{1}\right)=\left\{\begin{array}{l}
b_{21}(t) \text { if } t<t_{1}, \\
b_{22}(t) \text { if } t \geq t_{1} .
\end{array}\right.
$$

Given a commercialization time of player $1, t_{1}$, and a commercialization time of player $2, t_{2}$, the total benefits of the players are represented by the integrals

$$
\begin{aligned}
& B_{1}\left(t_{1}, t_{2}\right)=\int_{t_{1}}^{\infty} b_{1}\left(t \mid t_{2}\right) d t, \\
& B_{2}\left(t_{1}, t_{2}\right)=\int_{t_{2}}^{\infty} b_{2}\left(t \mid t_{1}\right) d t .
\end{aligned}
$$

Assumption 2.3. For every positive $t_{1}$ and every positive $t_{2}$ the integrals $B_{i}\left(t_{1}, t_{2}\right)(i=1,2)$ are finite. 
Given commercialization times of the players, $t_{1}$ and $t_{2}$, the total profit of player $i$ is defined as

$$
P_{i}\left(t_{1}, t_{2}\right)=-C_{i}\left(t_{i}\right)+B_{i}\left(t_{1}, t_{2}\right) .
$$

We define the game of timing for players 1 and 2 in line with the standards of game theory (see (Vorob'ev, 1977)). In the game of timing, the strategies of player $i(i=1,2)$ are the positive commercialization times, $t_{i}$, for project $i$, and the payoff to player $i$, thanks to strategies $t_{1}$ and $t_{2}$ of players 1 and 2 , respectively, is the total profit $P_{i}\left(t_{1}, t_{2}\right)$.

\section{NASH EQUILIBRIA}

According to the standard terminology of game theory, a strategy $t_{1}^{*}$ of player 1 is said to be a best response of player 1 to a strategy $t_{2}$ of player 2 if $t_{1}^{*}$ maximizes the payoff to player $1, P_{1}\left(t_{1}, t_{2}\right)$, over the set of all strategies of player $1, t_{1}$ :

$$
P_{1}\left(t_{1}^{*}, t_{2}\right)=\max _{t_{1}>0} P_{1}\left(t_{1}, t_{2}\right) .
$$

Similarly, a strategy $t_{2}^{*}$ of player 2 is said to be a best response of player 2 to a strategy $t_{1}$ of player 1 if $t_{2}^{*}$ maximizes the payoff to player $2, P_{2}\left(t_{1}, t_{2}\right)$, over the set of all strategies of player $2, t_{2}$ :

$$
P_{2}\left(t_{1}, t_{2}^{*}\right)=\max _{t_{2}>0} P_{2}\left(t_{1}, t_{2}\right) .
$$

Any pair $\left(t_{1}^{*}, t_{2}^{*}\right)$, where $t_{1}^{*}$ is a best response of player 1 to $t_{2}^{*}$ and $t_{2}^{*}$ is a best response of player 2 to $t_{1}^{*}$, is said to be a Nash equilibrium in the game of timing.

Our goal is to characterize the Nash equilibria in the game of timing. We start with analysis of the payoffs. The differentiation of $P_{1}\left(t_{1}, t_{2}\right)$ with respect to $t_{1}$ yields

$$
\begin{aligned}
\frac{\partial P_{1}\left(t_{1}, t_{2}\right)}{\partial t_{1}} & =a_{1}\left(t_{1}\right)-b_{1}\left(t_{1} \mid t_{2}\right) \\
& =\left\{\begin{array}{l}
a_{1}\left(t_{1}\right)-b_{11}\left(t_{1}\right) \text { if } t_{1}<t_{2}, \\
a_{1}\left(t_{1}\right)-b_{12}\left(t_{1}\right) \text { if } t_{1}>t_{2} .
\end{array}\right.
\end{aligned}
$$

Let us take two arbitrary strategies of player $2, t_{21}$ and $t_{22}>t_{21}$. As (9) shows,

$$
\frac{\partial P_{1}\left(t_{1}, t_{22}\right)}{\partial t_{1}}=\frac{\partial P_{1}\left(t_{1}, t_{21}\right)}{\partial t_{1}}
$$

for $t_{1}<t_{21}$ and for $t_{1}>t_{22}$, and

$$
\frac{\partial P_{1}\left(t_{1}, t_{22}\right)}{\partial t_{1}}=\frac{\partial P_{1}\left(t_{1}, t_{21}\right)}{\partial t_{1}}-\left(b_{11}\left(t_{1}\right)-b_{12}\left(t_{1}\right)\right) \text {, }
$$

for $t_{21}<t_{1}<t_{22}$. We have stated that beyond the time interval located between $t_{21}$ and $t_{22}, P_{1}\left(t_{1}, t_{22}\right)$ and $P_{1}\left(t_{1}, t_{21}\right)$ have the same rate in $t_{1}$, and within this interval $P_{1}\left(t_{1}, t_{22}\right)$ declines in $t_{1}$ faster than $P_{1}\left(t_{1}, t_{21}\right)$. Thanks to (6) and (5) $P_{1}\left(t_{1}, t_{22}\right)=$ $P_{1}\left(t_{1}, t_{21}\right)$ for $t_{1} \geq t_{22}$. Therefore, $P_{1}\left(t_{1}, t_{22}\right)>$ $P_{1}\left(t_{1}, t_{21}\right)$ for $t_{1}<t_{22}$.
Proposition 3.1. For every $t_{1}>0$, the payoff to player $1, P_{1}\left(t_{1}, t_{2}\right)$, increases in $t_{2}$; moreover, given a $t_{21}>0$ and a $t_{22}>t_{21}$, one has $P_{1}\left(t_{1}, t_{22}\right)=$ $P_{1}\left(t_{1}, t_{21}\right)$ for $t_{1} \geq t_{22}$, and $P_{1}\left(t_{1}, t_{22}\right)>P_{1}\left(t_{1}, t_{21}\right)$ for $t_{1}<t_{22}$.

Symmetric arguments are valid for player 2.

Proposition 3.2. For every $t_{2}>0$, the payoff to player $2, P_{2}\left(t_{1}, t_{2}\right)$, increases in $t_{1}$; moreover, given a $t_{11}>0$ and a $t_{12}>t_{11}$, one has $P_{2}\left(t_{12}, t_{2}\right)=$ $P_{2}\left(t_{11}, t_{2}\right)$ for $t_{2} \geq t_{12}$, and $P_{2}\left(t_{12}, t_{2}\right)>P_{2}\left(t_{11}, t_{2}\right)$ for $t_{2}<t_{12}$.

Let us find the best responses of player 1 to a given strategy, $t_{2}$, of player 2 . It is easy enough to identify the intervals of growth and decline of the payoff $P_{1}\left(t_{1}, t_{2}\right)$ as a function of $t_{1}$. We use (9) and refer to the points $t_{1}^{-}$and $t_{1}^{+}$, at which the graph of $a_{1}(t)$, intersects the graphs of $b_{11}(t)$ and $b_{12}(t)$.

Assume, first, that $t_{2} \leq t_{1}^{-}$; recall that $t_{1}^{-}<t_{1}^{+}$(see (4)). Then the graph of $a_{1}\left(t_{1}\right)$ lies above the graph of $b_{1}\left(t_{1} \mid t_{2}\right)$ for $t_{1}<t_{1}^{+}$and lies below it for $t_{1}>t_{1}^{+}$; at $t_{1}=t_{1}^{+}$the graphs intersect.

Due to (9), $\partial P_{1}\left(t_{1}, t_{2}\right) / \partial t_{1}$ is positive for $t_{1}<t_{1}^{+}$ $\left(t_{1} \neq t_{2}\right)$ and negative for $t_{1}>t_{1}^{+}$. Therefore, $t_{1}=t_{1}^{+}$ is the unique maximizer of $P_{1}\left(t_{1}, t_{2}\right)$ in the set of all positive $t_{1}$.

Let us assume that $t_{2} \geq t_{1}^{+}$. Then the graph of $a_{1}\left(t_{1}\right)$ lies above the graph of $b_{1}\left(t_{1} \mid t_{2}\right)$ for $t_{1}<t_{1}^{-}$, and lies below it for $t_{1}>t_{1}^{-}$; at $t_{1}=t_{1}^{+}$the graphs intersect. Due to (9), $\partial P_{1}\left(t_{1}, t_{2}\right) / \partial t_{1}$ is positive for $t_{1}<t_{1}^{-}$and negative for $t_{1}>t_{1}^{-}\left(t_{1} \neq t_{2}\right)$. Hence, $t_{1}=t_{1}^{-}$is the unique maximizer of $P_{1}\left(t_{1}, t_{2}\right)$ in the set of all positive $t_{1}$.

Now let $t_{2}$ lie in the interval $\left[t_{1}^{-}, t_{1}^{+}\right]$. Then the graph of $a_{1}\left(t_{1}\right)$ lies above the graph of $b_{1}\left(t_{1} \mid t_{2}\right)$ for $t_{1}<t_{1}^{-}$, lies below it for $t_{1}^{-}<t_{1}<t_{2}$, lies again above the graph of $b_{1}\left(t_{1} \mid t_{2}\right)$ for $t_{2}<t_{1}<t_{1}^{+}$and again below it for $t_{1}>t_{1}^{+}$. Thanks to (9) we conclude that, $P_{1}\left(t_{1}, t_{2}\right)$, as a function of $t_{1}$, strictly increases on the interval $\left(0, t_{1}^{-}\right)$, strictly decreases on the interval $\left(t_{1}^{-}, t_{2}\right)$, strictly increases on the interval $\left(t_{2}, t_{1}^{+}\right)$, and strictly decreases on the interval $\left(t_{1}^{+}, \infty\right)$. Therefore, the maximizers of $P_{1}\left(t_{1}, t_{2}\right)$ in the set of all positive $t_{1}$ are restricted to the two-element set $\left\{t_{1}^{-}, t_{1}^{+}\right\}$.

Let us identify the actual maximizers in this set. Suppose $t_{2}<t_{1}^{+}$. Set $t_{1}=t_{1}^{+}, t_{21}=t_{2}$ and $t_{22}=t_{1}^{+}$. We see that $t_{1}=t_{22}>t_{21}$. By Proposition 3.1 we have

$$
P_{1}\left(t_{1}^{+}, t_{1}^{+}\right)=P_{1}\left(t_{1}^{+}, t_{2}\right) .
$$

Since $P_{1}\left(t_{1}^{+}, t_{2}\right)$ is continuous in $t_{2},(10)$ holds for $t_{2}=t_{1}^{+}$. Now we take arbitrary $t_{21}$ and $t_{22}>t_{21}$ in the interval $\left[t_{1}^{-}, t_{1}^{+}\right]$. By Proposition 3.1 $P_{1}\left(t_{1}^{-}, t_{22}\right)$ $>P_{1}\left(t_{1}^{-}, t_{21}\right)$. Therefore, $P_{1}\left(t_{1}^{-}, t_{2}\right)$ strictly increases in $t_{2}$ on $\left[t_{1}^{+}, t_{2}^{+}\right]$. Consider the function

$$
p\left(t_{2}\right)=P_{1}\left(t_{1}^{-}, t_{2}\right)-P_{1}\left(t_{1}^{+}, t_{2}\right),
$$

defined on $\left[t_{1}^{-}, t_{1}^{+}\right]$. By (10) we have 


$$
p\left(t_{2}\right)=P_{1}\left(t_{1}^{-}, t_{2}\right)-P_{1}\left(t_{1}^{+}, t_{1}^{+}\right),
$$

for all $t_{2}$ in the interval $\left[t_{1}^{+}, t_{2}^{+}\right]$. As long as $P_{1}\left(t_{1}^{-}, t_{2}\right)$ strictly increases in $t_{2}$ on $\left[t_{1}^{-}, t_{1}^{+}\right], p\left(t_{2}\right)$ strictly increases on $\left[t_{1}^{+}, t_{2}^{+}\right]$. Earlier, we have stated that $t_{1}^{+}$ is the single best response of player 1 to any $t_{2} \leq t_{1}^{-}$; this holds, in particular, for $t_{2}=t_{1}^{-}$, i.e.,

$$
P_{1}\left(t_{1}^{+}, t_{1}^{-}\right)>P_{1}\left(t_{1}^{-}, t_{1}^{-}\right) \text {. }
$$

Hence,

$$
p\left(t_{1}^{-}\right)=P_{1}\left(t_{1}^{-}, t_{1}^{-}\right)-P_{1}\left(t_{1}^{+}, t_{1}^{-}\right)<0 .
$$

Earlier, we have stated that $t_{1}^{-}$is the single best response of player 1 to any $t_{2} \geq t_{1}^{+}$; this holds, in particular, for $t_{2}=t_{1}^{+}$, i.e.,

$$
P_{1}\left(t_{1}^{-}, t_{1}^{+}\right)>P_{1}\left(t_{1}^{+}, t_{1}^{+}\right) .
$$

Hence,

$$
p\left(t_{1}^{+}\right)=P_{1}\left(t_{1}^{-}, t_{1}^{+}\right)-P_{1}\left(t_{1}^{+}, t_{1}^{+}\right)>0 .
$$

We have found that $p\left(t_{2}\right)$ takes a negative value at the left end point of the interval $\left[t_{1}^{-}, t_{1}^{+}\right]$and a positive value at the right end point of this interval. Since $p\left(t_{2}\right)$ is continuous, there exists a $\hat{t}_{2}$ in the interior of $\left[t_{1}^{-}, t_{2}^{+}\right]$, for which $p\left(\hat{t}_{2}\right)=0$. The fact that $p\left(t_{2}\right)$ strictly increases on $\left[t_{1}^{-}, t_{1}^{+}\right]$implies that the point $t_{2}$ is unique, i.e., $p\left(t_{2}\right)<0$ for $t_{1}^{-} \leq t_{2}<\vec{t}_{2}$ and $p\left(t_{2}\right)>0$ for $t_{1}^{+} \geq t_{2}>\hat{t}_{2}$. By the definition of $p\left(t_{12}\right)$, (11), we have

$$
\begin{gathered}
P_{1}\left(t_{1}^{-}, \hat{t}_{2}\right)=P_{1}\left(t_{1}^{+}, \hat{t}_{2}\right), \\
P_{1}\left(t_{1}^{-}, t_{2}\right)<P_{1}\left(t_{1}^{+}, t_{2}\right) \text { for } t_{1}^{-} \leq t_{2}<\hat{t}_{2}, \\
P_{1}\left(t_{1}^{-}, t_{2}\right)<P_{1}\left(t_{1}^{+}, t_{2}\right) \text { for } t_{1}^{+} \geq t_{2}>\hat{t}_{2} .
\end{gathered}
$$

All best responses of player 1 to $t_{2}$ lie in the twoelement set $\left\{t_{1}^{-}, t_{1}^{+}\right\}$. Therefore, we conclude that if $t_{2}=\hat{t}_{2}$, player 1 has two best responses, $t_{1}^{-}$and $t_{1}^{+}$, to $t_{2}$; if $t_{1}^{-} \leq t_{2}<\hat{t}_{2}$, the unique best response of player 1 to $t_{2}$ is $t_{1}^{+}$; and if $t_{1}^{+} \geq t_{2}>\hat{t}_{2}$, the unique best response of player 1 to $t_{2}$ is $t_{1}^{-}$. The best response of player 1 to $t_{2}$ is $t_{1}^{+}$if $t_{2}<t_{1}^{-}$, and $t_{1}^{-}$if $t_{2}>t_{1}^{+}$. We summarize as follows.

Proposition 3.3. In the interval $\left(t_{1}^{-}, t_{1}^{+}\right)$, there exists the unique point $\hat{t}_{2}$ such that

$$
P_{1}\left(t_{1}^{-}, \hat{t}_{2}\right)=P_{1}\left(t_{1}^{+}, \hat{t}_{2}\right) \text {. }
$$

The set of all best responses of player 1 to $\hat{t}_{2}$ is $\left\{t_{1}^{-}, t_{1}^{+}\right\}$. If $0<t_{2}<\hat{t}_{2}$, then the unique best response of player 1 to $t_{2}$ is $t_{1}^{+}$. If $t_{2}>\hat{t}_{2}$, then the unique best response of player 1 to $t_{2}$ is $t_{1}^{-}$.

We call $t_{1}^{-}$the fast choice of player 1 and $t_{1}^{+}$the slow choice of player 1 . We call $\hat{t}_{2}$ the switch point for player 1 .

Let us consider the function that associates to each strategy, $t_{2}$, of player 2 the set of all best responses of player 1 to $t_{2}$; we call it the best response function of player 1 . The graph of the best response function of player 1 consists of the horizontal segment located strictly above the segment $\left(0, \hat{t}_{2}\right]$ on the $t_{2}$ - axis at level $t_{1}^{+}$, and the unbounded horizontal segment located strictly above the segment $\left[\hat{t}_{2}, \infty\right)$ on the $t_{2}$ axis at level $t_{1}^{-}$. Points $\left(t_{1}^{+}, \hat{t}_{2}\right)$ and $\left(t_{1}^{-}, \hat{t}_{2}\right)$ lie on the graph.

A symmetric argument characterizes the best responses of player 2 .

Proposition 3.4. In the interval $\left(t_{2}^{-}, t_{2}^{+}\right)$, there exists the unique point $\tilde{t}_{1}$ such that

$$
P_{2}\left(\hat{t}_{1}, t_{2}^{-}\right)=P_{1}\left(\hat{t}_{1}, t_{2}^{+}\right) .
$$

The set of all best responses of player 2 to $\hat{t}_{1}$ is $\left\{t_{2}^{-}, t_{2}^{+}\right\}$. If $0<t_{1}<\hat{t}_{1}$, then the unique best response of player 2 to $t_{1}$ is $t_{2}^{+}$. If $t_{1}>\hat{t}_{1}$, then the unique best response of player 2 to $t_{1}$ is $t_{2}^{-}$.

We call $t_{2}^{-}$the fast choice of player $2, t_{2}^{+}$the slow choice of player 2 , and $\hat{t}_{2}$ the switch point for player 2 . We also introduce the best response function of player 2 , which associates to each strategy, $t_{1}$, of player 1 the set of all best responses of player 2 to $t_{1}$. The graph of the best response function of player 2 consists of the vertical segment located to the right of the segment $\left(0, \hat{t}_{1}\right]$ on the $t_{1}$ - axis at distance $t_{2}^{+}$, and the unbounded vertical segment located to the right of the segment $\left[\hat{t}_{1}, \infty\right)$ on the $t_{1}$ - axis at distance $t_{2}^{-}$. Points $\left(\hat{t}_{1}, t_{2}^{+}\right)$and $\left(\hat{t}_{1}, t_{2}^{-}\right)$lie on the graph.

Nash equilibria $\left(t_{1}^{*}, t_{2}^{*}\right)$ belong to the intersection of the graphs of the best response functions of players 1 and 2 and are characterized by the following relations

$$
\begin{gathered}
\hat{t}_{2} \geq t_{2}^{+}, \quad \hat{t}_{1}<t_{1}^{+}, \\
\hat{t}_{2} \geq t_{2}^{+}, \quad t_{1}^{-}<\hat{t}_{1}<t_{1}^{+}, \\
\hat{t}_{2} \leq t_{2}^{-}, \quad t_{1}^{-}<\hat{t}_{1}<t_{1}^{+}, \\
t_{2}^{-} \leq \hat{t}_{2}<t_{2}^{+}, \quad t_{1}^{-}<\hat{t}_{1} \leq t_{1}^{+}, \\
t_{2}^{-}<\hat{t}_{2} \leq t_{2}^{+}, \quad t_{1}^{-} \leq \hat{t}_{1}<t_{1}^{+}, \\
t_{2}^{-}<\hat{t}_{2}<t_{2}^{+}, \quad \hat{t}_{1} \leq t_{1}^{-}, \\
t_{2}^{-}<\hat{t}_{2}<t_{2}^{+}, \quad \hat{t}_{1} \geq t_{1}^{+}, \\
\hat{t}_{2}<t_{2}^{-}, \quad \hat{t}_{1} \geq t_{1}^{+} .
\end{gathered}
$$

Proposition 3.5. In cases (14), (15) and (19) the unique Nash equilibrium is slow-fast, $\left(t_{1}^{-}, t_{2}^{+}\right)$. In cases (16), (20) and (21) the unique Nash equilibrium is fast-slow, $\left(t_{1}^{+}, t_{2}^{-}\right)$. In cases (17) and (18) the game of timing has precisely two Nash equilibria, fast-slow, $\left(t_{1}^{-}, t_{2}^{+}\right)$, and slow-fast, $\left(t_{1}^{+}, t_{2}^{-}\right)$.

Proposition 3.6. Let the game of timing have two Nash equilibria, i.e., (17) or (18) hold. Then

(i) $P_{1}\left(t_{1}^{-}, t_{2}^{+}\right) \geq P_{1}\left(t_{1}^{+}, t_{2}^{-}\right)$, moreover, the inequality is strict if and only if $\hat{t}_{2}<t_{2}^{+}$;

(ii) $P_{2}\left(t_{1}^{-}, t_{2}^{+}\right) \geq P_{2}\left(t_{1}^{+}, t_{2}^{-}\right)$, moreover, the inequality is strict if and only if $\hat{t}_{1}<t_{1}^{+}$.

We conclude the general part of our study with the description of an algorithm that finds the Nash equilibria in the game of timing.

Step 1. Use definitions for finding the players' fast and slow choices, $t_{i}^{-}, t_{2}^{+}(i=1,2)$. 
Step 2. Use definitions (12) and (13) for finding the players' switch times, $\hat{t}_{i}(i=1,2)$.

Step 3. Use Proposition 3.5 for identifying the Nash equilibria.

\section{GAS PIPELINE GAME}

We apply the suggested solution method to a model described in (Klaassen et al., 2001b).

The cost $C_{i}\left(t_{i}\right)$ for finalizing the construction of pipeline $i(i=1,2)$ at time $t_{i}$ is defined to be the minimum of the integral investments

$$
I_{i}\left(r_{i}\right)=\int_{0}^{t_{i}} e^{-\lambda t} r_{i}(t) d t .
$$

Here $\lambda$ is a positive discount. An investment strategy of player $i$ is modeled as an integrable control function,

$$
r_{i}(t)>0,
$$

that brings the accumulated investment, $x_{i}(t)$, from 0 to the prescribed commercialization level $\bar{x}_{i}>0$ at time $t_{i}$.

The dynamics of $x_{i}(t)$ is modeled as

$$
\dot{x}_{i}(t)=-\sigma x_{i}(t)+r_{i}^{\gamma}(t) .
$$

Here $\sigma$ is a positive obsolescence coefficient and $\gamma$ $(0<\gamma<1)$ is a delay parameter.

In the supply game arising at time $t$, the strategies of player $i$ are nonnegative rates of supply, $y_{i}$, and the payoff to player $i$ is defined as

$$
p_{i}\left(y_{1}, y_{2} \mid t\right)=e^{-\lambda t}\left(\pi(t, y)-c_{i}(t)\right) y_{i} .
$$

Here $y$ is the total rate of supply, $\pi(t, y)$ is the price of gas and $c_{i}(t)>0$ is the cost for extraction and transportation of gas for player $i$. The price of gas is modeled as

$$
\pi(y \mid t)=\left(\frac{g(t)}{y}\right)^{\beta},
$$

where $g(t)>0$ is the consumer's GDP at time $t$ and $\beta$ $(0<\beta<1)$ is the inverse to the price elasticity of gas demand.

Assumption 5.1. Assume that the following conditions hold

$$
1-\frac{(2-\beta) c_{i}(t)}{c_{1}(t)+c_{2}(t)}>0 \quad(i=1,2) .
$$

Proposition 5.1. For player $i(i=1,2)$ the following formulas hold.

1. The cost, $C_{i}\left(t_{i}\right)$, is given

$$
C_{i}\left(t_{i}\right)=\rho^{\alpha-1} \frac{e^{-\lambda t_{i}} \bar{x}_{i}^{\alpha}}{\left(1-e^{-\rho t_{i}}\right)^{\alpha-1}},
$$

where

$$
\alpha=\frac{1}{\gamma}, \quad \rho=\frac{\alpha \sigma+\lambda}{\alpha-1}
$$

2. The rate of cost reduction, $a_{i}\left(t_{i}\right)$, is given by

$$
a_{i}(t)=\rho^{\alpha-1} \bar{x}_{i}^{\alpha} \frac{e^{-\lambda t}\left(\lambda+\nu e^{-\rho t}\right)}{\left(1-e^{-\rho t}\right)^{\alpha}},
$$

where

$$
\nu=\alpha \sigma \text {. }
$$

3. The upper benefit rate, $b_{i 1}\left(t_{1}\right)$, is given by

$$
b_{i 1}(t)=e^{-\lambda t}(1-\beta)^{1 / \beta-1} \frac{g(t)}{c_{i}^{1 / \beta-1}(t)} .
$$

4. The lower benefit rate, $b_{i 2}\left(t_{i}\right)$, is given by

$$
\begin{aligned}
& b_{i 2}(t)=e^{-\lambda t}(2-\beta)^{1 / \beta-1}\left(1-\frac{(2-\beta) c_{i}(t)}{c_{1}(t)+c_{2}(t)}\right)^{2} \\
& \times \frac{g(t)}{\left(c_{1}(t)+c_{2}(t)\right)^{1 / \beta-1}} .
\end{aligned}
$$

5 . The following inequality is valid

$$
b_{i 1}(t)>b_{i 2}(t) \text {. }
$$

Assumption 5.2. Assume that the consumer's GDP, $g(t)$, and costs, $c_{i}(t)$, grow exponentially,

$$
g(t)=g^{0} e^{\zeta t}, \quad c_{i}(t)=c_{i}^{0} e^{\omega t}(i=1,2),
$$

( $\zeta$ and $\omega$ are nonnegative), and

$$
0<\kappa<\lambda \text {, }
$$

where

$$
\kappa=\zeta-\left(\frac{1}{\beta}-1\right) \omega .
$$

Proposition 5.2. Under assumptions 5.1 and 5.2 the fast choice, $t_{i}^{-}$, and the slow choice, $t_{i}^{+}$, of player $i(i=1,2)$ is the unique solution of the algebraic equation

$$
e^{-\lambda t} \rho^{\alpha-1} \bar{x}_{i}^{\alpha}=\frac{\left(1-e^{-\rho t}\right)^{\alpha}}{\lambda+\nu e^{-\rho t}} b_{i j}(t) .
$$

for $j=1,2$ respectively.

We assume that $g(t)$ and $c_{i}(t)(i=1,2)$ are given by (33) and inequality (34) is satisfied. Formulas (30) and (31) for $b_{i 1}(t)$ and $b_{i 2}(t)$ are specified as

$$
b_{i 1}(t)=b_{i 1}^{0} e^{-\psi t}, \quad b_{i 2}(t)=b_{i 2}^{0} e^{-\psi t},
$$

where

$$
\psi=\lambda-\kappa, b_{i 1}^{0}=b_{i 1}\left(t_{0}\right), b_{i 2}^{0}=b_{i 2}\left(t_{0}\right) .
$$

Proposition 5.3. For $i=1,2$ we have

$$
\hat{t}_{i}=-\frac{1}{\psi} \log \left(\frac{\psi G_{i}}{b_{i 2}^{0}-b_{i 1}^{0}}\right),
$$

where

$$
\begin{aligned}
& G_{i}=-\frac{\rho^{\alpha-1} e^{-\lambda t_{i}^{+}} \bar{x}_{1}^{\alpha}}{\left(1-e^{-\rho t_{1}^{+}}\right)^{\alpha-1}}+\frac{b_{i 2}^{0} e^{-\psi t_{i}^{+}}}{\psi}+ \\
& \frac{\rho^{\alpha-1} e^{\lambda t_{i}^{-}} \bar{x}_{1}^{\alpha}}{\left(1-e^{-\rho t_{i}^{-}}\right)^{\alpha-1}}-\frac{b_{i 1}^{0} e^{-\psi t_{i}^{-}}}{\psi} .
\end{aligned}
$$




\section{CASE STUDY}

We consider the game of timing in application to the Caspian and China gas markets. The values of the model parameters are based on preliminary expert estimates. Our first case study deals with the competition of two major gas pipeline projects in the Caspian region, the "Blue Stream" project of the Russian GAZPROM Company (project 1) which is aimed at delivering Russian gas to Turkey under the Black Sea; and the "Trans-Caspian" project (project 2) directed from Turkmenistan underneath the Caspian Sea through Azerbaijan and Georgia to Turkey. In this case study the parameters of the model are chosen as follows - the discount rate: $\lambda=0.1$; the obsolescence coefficient: $\sigma=0.3$; the delay coefficient: $\gamma=0.65$; the inverse to the price elasticity of gas demand: $\beta=0.55$; the initial level of the consumer's GDP: $g^{0}=214.6$; the growth rate of the consumer's GDP: $\zeta=0.1$; the growth rate of the extraction costs: $\omega=0.15$; the initial extraction costs: $c_{1}^{0}=67.3$, $c_{2}^{0}=78.4$; the commercialization levels of the accumulated investments: $\bar{x}_{1}=4.0, \bar{x}_{2}=2.5$. It is assumed that the projects start in 2001.

For these parameters there exist two Nash equilibria in the game of timing, the fast-slow equilibrium $\left(t_{1}^{-}, t_{2}^{+}\right)=(2002.8,2005.2)$, and the slow-fast equilibrium $\left(t_{1}^{+}, t_{2}^{-}\right)=(2004.6,2002.2)$.

Our second case study is related to the planned projects of gas pipelines from Russia to China. Two potential competitors on the North China gas market are the "Kovikta-Zabaikalsk-Kharbin" pipeline (project 1) stretched from the Irkutsk region to North China, and the "Sakhalin-Khabarovsk-Kharbin" pipeline (project 2). The following values of the model parameters are chosen: $\lambda=0.1, \sigma=0.3, \gamma=0.58$, $\beta=0.46, g^{0}=1157, \zeta=0.0668, \omega=0.05$, $c_{1}^{0}=57, c_{2}^{0}=68, \bar{x}_{1}=6, \bar{x}_{2}=3$. The initial year for the projects is set in 2001 .

In this case study there exists the unique, slow-fast, Nash equilibrium $\left(t_{1}^{+}, t_{2}^{-}\right)=(2003.6,2002)$.

\section{CONCLUSIONS}

The paper is devoted to the analysis of a twoplayer game, in which the players' strategies are times of terminating innovation processes. In the game between the projects the total profits act as payoffs and commercialization times as strategies. The analysis of the game leads to the restriction of player's rational choices to no more than two prescribed combinations of commercialization times, which constitute the Nash equilibria in the game. Typically, two Nash equilibria arise and the projects compete for a fast commercialization scenario; its complement, a slow commercialization scenario, is less profitable, representing the best response to the fast scenario of the competitor. An algorithm for finding the Nash equilibria is described.

\section{REFERENCES}

Arrow, K.J. and M. Kurz (1970). Public Investment, the Rate of Return and Optimal Fiscal Policy. Johns Hopkins University Press. Baltimore.

Barzel, Y. (1968). Optimal timing of innovations. The Review of Economics and Statistics 50, 348-355.

Basar, T. and G.J. Olsder (1982). Dynamic Noncooperative Game Theory. Academic Press. London, New York.

Chernousko, F.L. (1994). State Estimation for Dynamic Systems. CRC Press. Boca Raton, Florida.

Friedman, D. (1991). Evolutionary games in economics. Econometrica 59, 637-666.

Hofbauer, J. and K. Sigmund (1988). The Theory of Evolution and Dynamic Systems. Cambridge University Press. Cambridge.

Intriligator, M. (1971). Mathematical Optimization and Economic Theory. Prentice-Hall. New York.

Kaniovski, Yu.M., A.V. Kryazhimskii and H.P. Young (2000). Adaptive dynamics in games played by heterogeneous populations. Games and Economic Behavior 31, 50-96.

Klaassen, G., A. McDonald and J. Zhao (2001a). The future of gas infrastructures in Eurasia. Energy Policy 29, 399-413.

Klaassen, G., R.A. Roehrl and A.M. Tarasyev (2001b). The great Caspian pipeline game. IIASA IR-0166, 107-131.

Krasovskii, N.N. and A.I. Subbotin (1988). GameTheoretical Control Problems. Springer. New York, Berlin.

Kryazhimskii, A., A. Nentjes, S. Shibayev and A. Tarasyev (2001). Modeling market equilibrium for transboundary environmental problem. Nonlinear Analysis: Theory, Methods and Applications 42, 991-1002.

Kryazhimskii, A.V. and Yu.S. Osipov (1995). On evolutionary-differential games. Proceedings of Steklov Institute of Mathematics 211, 257-287.

Pontryagin, L.S., V.G. Boltyanskii, R.V. Gamkrelidze and E.F. Mishchenko (1962). The Mathematical Theory of Optimal Processes. Interscience. NY.

Tarasyev, A.M. (1999). Control synthesis in grid schemes for Hamilton-Jacobi equations. Annals of Operations Research 88, 337-359.

Tarasyev, A.M. and C. Watanabe (2001). Dynamic optimality principles and sensitivity analysis in models of economic growth. Nonlinear Analysis: Theory, Methods and Applications 47, 23092320.

Vorob'ev, N.N. (1977), Game Theory Lectures for Economists and Systems Scientists. Springer. New York, Berlin. 